\title{
Patient and public involvement in clinical guidelines: international experiences and future perspectives
}

\author{
Antoine Boivin, ${ }^{1}$ Kay Currie, ${ }^{2}$ Béatrice Fervers, ${ }^{3}$ Javier Gracia, ${ }^{4}$ Marian James, ${ }^{5}$ \\ Catherine Marshall, ${ }^{6}$ Carol Sakala, ${ }^{7}$ Sylvia Sanger, ${ }^{8}$ Judi Strid, ${ }^{9}$ Victoria Thomas, ${ }^{10}$ \\ Trudy van der Weijden, ${ }^{11}$ Richard Grol, ${ }^{1}$ Jako Burgers, ${ }^{1}$ on behalf of G-I-N PUBLIC
}

\begin{abstract}
${ }^{1}$ Scientific Institute for Quality of Healthcare, Nijmegen, The Netherlands

${ }^{2}$ National Health \& Medical Research Council, Canberra, Australia

${ }^{3}$ Centre Léon Bérard, Université de Lyon, Lyon, France

${ }^{4}$ Unidad de Evaluación de Tecnologías Sanitarias, Madrid, Spain

${ }^{5}$ Agency for Healthcare Research and Quality, Rockville, MD, USA

${ }^{6}$ Independent Guideline Advisor, Waipukurau, New Zealand

${ }^{7}$ Childbirth Connection, New York, NY, USA

${ }^{8}$ Agency for Quality in Medicine, Berlin, Germany

${ }^{9}$ National Consumer Advocacy Service, Auckland, New Zealand

${ }^{10}$ National Institute for Health and Clinical Excellence, London, UK

${ }^{11}$ Maastricht University, Maastricht, The Netherlands
\end{abstract}

Correspondence to Dr Antoine Boivin, Scientific Institute for Quality of Healthcare, Radboud University Nijmegen Medical Centre, Nijmegen, The Netherlands; antoine.boivin@gmail.com

Accepted 14 November 2009 Published Online First 27 April 2010

\section{ABSTRACT}

Background Clinical practice guidelines (CPG) are important tools for improving patient care. Patient and public involvement is recognised as an essential component of CPG development and implementation. The Guideline International Network Patient and Public Involvement Working Group (G-I-N PUBLIC) aims to support the development, implementation and evaluation of guideline-oriented patient and public involvement programmes (PPIPS).

Objective To develop an international practice and research agenda on patient and public involvement in CPG.

Method 56 CPG developers, researchers, and patient/ public representatives from 14 different countries, were consulted in an international workshop.

Recommendations were validated with G-I-N PUBLIC steering committee members.

Results Many CPG organisations have set up PPIPs that use a range of participation, consultation and communication methods. Current PPIPs aim to improve the quality and responsiveness of CPGs to public expectations and needs, or to foster individual healthcare decisions. Some organisations use structured involvement methods, including providing training for patient and public representatives. A number of financial, organisational and sociopolitical barriers limit patient and public involvement. The paucity of process and impact evaluations limits our current understanding of the conditions under which patient and public involvement is most likely to be effective.

Conclusion Greater international collaboration and research are needed to strengthen existing knowledge, development and evaluation of patient and public involvement in CPG.

\section{INTRODUCTION}

Clinical practice guidelines (CPGs) are important tools for improving patient care. ${ }^{12}$ Many national organisations and experts recommend involving patients, consumers and the public in CPG development and implementation. ${ }^{3-6}$ In recent years, patient and public involvement programmes (PPIPs) have been developed with wide variations between countries. This partly reflects the thin evidence guiding the development of PPIP $^{4}{ }^{7}$ but also highlights how cultural, organisational context, and stakeholders' perspectives influence these programmes. ${ }^{8}$

In 2007, the Guideline International Network ${ }^{9}$ created its Patient and Public Involvement Working Group (G-I-N PUBLIC, http://www. ginpublic.net). G-I-N PUBLIC aims to support effective patient and public involvement in specific contexts of CPG development and implementation through: the sharing of experiences and evidence, international research collaboration, and the development of standards and methods of involvement. To date, no structured consultation process has been used to establish priorities in the field. This paper presents current international experiences, and a research and practice agenda for the development of effective patient and public involvement in CPG, based on the results of an international workshop organised by G-I-N PUBLIC.

\section{METHODS}

\section{Definitions and scope}

Different labels are used internationally to describe involvement methods and its participants (consumers, users, citizens, patients and the public).$^{10}$ For the purpose of the workshop, PPIP refers to at least one formal method of involving patients and the public in CPG development and/or implementation. Involvement methods may include: communication (information is communicated to patients/the public); consultation (information is collected from patients/the public); or participation (patients/the public exchange information with other stakeholders) ${ }^{11}$ PPIP can be used at different stages, from the macro-level of CPG development (eg, topic selection, evidence review, recommendation, and development of ancillary products), its meso-level of implementation to specific target groups, or its use at the micro-level of the clinical consultation.

\section{International consultation and validation}

G-I-N PUBLIC members organised a $3 \mathrm{~h}$ interactive workshop $^{12} 13$ using structured and facilitated discussion at the 5th Guidelines International Network conference in Helsinki (Finland), in November 2008. Participants were divided into subgroups to foster active participation and to address specific questions, including (1) expectations and goals of PPIP; (2) participation and consultation in CPG development; (3) the integration of patient decision support technologies; (4) priorities for research and international collaboration. In each subgroup, a facilitator introduced the topic and chaired discussions, while a different person took notes. An oral summary of discussions was fed back to all participants at the end of the workshop, and facilitators produced a written report of discussions. ${ }^{14}$ We distributed the original data to 
G-I-N PUBLIC Steering Committee members and solicited written comments to validate findings, before discussing recommendations with them in two teleconferences.

\section{RESULTS}

A total of 56 people from 14 countries participated in the workshop and subsequent discussions (table 1). Results are reported as per the main themes of discussion presented above.

\section{Expectations and goals of PPIP}

Workshop participants agreed on the importance of patient and public involvement in CPG. However, they reported that patient and public involvement goals are often largely implicit or articulated in vague terms by their organisations, which makes it difficult to assess success or failure. Tension also exists between collective and individual perspectives. From a collective perspective, PPIP can be seen as a way to develop recommendations that will improve the quality of healthcare and its responsiveness to population needs and expectations. Patient and public involvement can also be seen as an accountability mechanism that fosters CPG social legitimacy and its ability to be implemented in clinical practice. At the individual level, patient and public involvement can be geared towards the promotion of individuals' rights and the protection of patients' autonomy and freedom of choice. For some, the position of patients as consumers and users of healthcare justified their participation in CPG development. Other PPIP goals discussed by participants were focused on promoting informed choice to ensure that patient/provider interaction was patient-centred and responsive to individual needs, values and priorities. Participants considered that differences in perspectives can have a profound impact on the choice of methods for involving patients and the public, as well as on the criteria used to assess PPIP effectiveness.

\section{Participation and consultation in CPG development}

Participants agreed that participation methods, where patient or public representatives exchange information and deliberate with other CPG developers, are present in many existing PPIPs. CPG organisations often include patient members in guideline development groups to provide consumer perspectives in the interpreting of the evidence and develop recommendations that

Table 1 Details of participants

\begin{tabular}{ll}
\hline & $\begin{array}{l}\text { Participants } \\
\mathbf{n = 5 6}\end{array}$ \\
\hline Role in relation to guidelines & \\
Guideline developers & 35 \\
Researchers & 16 \\
Patient and public representatives & 5 \\
Country (n=14) & \\
Australia & 3 \\
Belgium & 2 \\
Canada & 5 \\
Czech Republic & 3 \\
Finland & 5 \\
France & 6 \\
Germany & 3 \\
Japan & 4 \\
The Netherlands & 8 \\
New Zealand & 2 \\
Norway & 5 \\
Spain & 4 \\
UK & 3 \\
USA & 3 \\
\hline
\end{tabular}

are relevant to patients. The National Institute for Health and Clinical Excellence (NICE) citizens' council uses deliberative participation methods to involve members of the general public to discuss social values related with CPG development.

Participants pointed towards the importance of recruitment, support and training as key conditions for meaningful involvement of patient and public representatives. Training may cover the fundamentals of guideline development and approaches for reporting back to consumer constituencies, or offer mentoring opportunities from other patient/public representatives. Participants concluded that training and support may facilitate understanding of the technical aspects of CPG development, address financial and organisational barriers to participation, and enhance mutual understanding regarding the role of PPIP.

Participants highlighted the importance of recruiting patient/ public members early in the process, with consideration given to a balanced socio-demographic representation, because many CPG disproportionately impact certain subgroups. A key recruitment question is whether patient/public members should be expected to represent a constituency or to bring their personal experience to the table: job descriptions are used by some participants' organisations and may assist in clarifying expectations. Concerns were raised about the dependence of some consumer organisations on pharmaceutical or biomedical industry funding and its potential impact on CPG validity: some participants reported that their organisation required declarations of interests from all CPG developers, including patients/ public members. On the other hand, patient representatives argued that some patients' and consumers' organisations have became quite sophisticated in their understanding of the evidence, as exemplified by the Cochrane Consumer Network, and that their involvement could strengthen the quality of CPG by expanding the range of evidence being considered and by questioning certain experts' assumptions.

Participants regarded open consultation and written submissions by patient organisations as particularly useful in defining CPG topics and comments on draft CPG. Participants reported that the use of a focus group has been useful at the beginning of the CPG development process, when little evidence on patients' preferences is available, or at the end of the process to test recommendations and improve its potential for implementation. Participants also noted that little is done currently to synthesise existing published evidence on patient and public views and preferences, and suggested that the range of consultation methods currently used in PPIP could be expanded to include satisfaction surveys, or web-based consultations.

\section{Integration of patient decision support technologies}

According to workshop participants, many PPIP focus on communication methods to promote more active and informed health decisions by patients and consumers at the micro-level of the clinical consultations. Some organisations (eg, German Agency for Quality in Medicine, New-Zealand Guideline Group and NICE) have developed large online repositories and short summaries of patient versions of CPG. Others have attempted to disseminate CPGs through collaboration with patient organisations. ${ }^{15}$ Participants agreed that the development of patient oriented material may assist understanding of CPG recommendations and could foster informed and shared decision-making between patients and clinicians. For example, the Dutch Institute for Healthcare Improvement (CBO) has produced patient decision aids presenting options, individualised risk assessment, and the probabilities of benefits and downsides to support choices regarding 'preference sensitive decisions' (such as 
prostate cancer screening), ${ }^{16}$ which are characterised either by evidence that points to a balance between harms and benefits or by scientific uncertainty. ${ }^{17}$

Participants suggested that professional versions of CPG could also be adapted to foster shared decision-making. For example, methods could be developed to search and analyse preferencerelated evidence and present it in the CPG. Some guideline organisations also convey information about the relative importance of interventions for patients in their grading of recommendations. It was also felt that professional versions of CPG could signal decisions and recommendations that are most likely to require discussion with patients, decision-support tools, or specific preference-eliciting and communication strategies. With the growth of increasingly sophisticated health information technology, guidelines and decision tools can be disseminated in concert (eg, by hypertext links in electronic guideline documents), rather than as stand-alone products.

\section{Research agenda and the role of international collaboration}

Participants identified priorities for research and international collaboration (table 2). They indicated the lack of evaluation of PPIP as a barrier to the wider acceptance and development of PPIP. Participants identified diverging roles for evaluation: for some, research should help to assess the added value of PPIP in CPG development and implementation, while others see involvement as having intrinsic value, and evaluation efforts are expected to help develop and identify the most effective involvement methods.

There was a high demand among participants for stronger international collaboration on patient and public involvement within and outside the CPG community. Participants saw the international community as a pool of ideas and experiences, and expressed the need to share both 'success' and 'failure' stories. The heterogeneous level of expertise and capacity, as well as cultural and health system variations, was seen as both a motivator and a barrier for greater international collaboration.

\section{DISCUSSION}

To our knowledge, this paper reports the first international consultation to develop priorities for research and collaboration on patient and public involvement in CPG, based on international experience. The involvement of patients and the public is motivated by attempts to improve CPG quality and implementation, and its responsiveness to population expectations and needs, and to foster individual healthcare choices. ${ }^{8} 1819$ Current approaches favour involvement of patient members on guideline development groups, consultation of draft CPG and the development of patient versions of CPG, an observation that is in line with previous surveys of CPG organisations. ${ }^{20-22}$ The range of reported methods to involve patients and the public appears to make little use of alternative methods proposed in the literature, such as systematic reviews of published evidence on patients' views and preferences, the integration of patient decision aids, the use of decision analysis to integrate patients' utilities in CPG recommendations and their involvement in strategic aspects of CPG development, including CPG evaluation. $^{4} 1623-30$

Previous reviews have found few empirical studies of PPIP in the context of CPG development or implementation. ${ }^{7}$ 31-34 The evidence supporting when and how PPIP should be developed needs to be strengthened. ${ }^{35}$ These efforts should recognise the specific challenges of evaluating patient and public involvement, including: (1) the absence of consensus over what constitutes 'effective involvement'; (2) the lack of agreed-upon evaluation tools; and (3) the value-laden and context-sensitive nature of PPIP. ${ }^{36} 37$

The strength of this study rests on the broad reach of the consultation process, with direct participation of 56 representatives from 14 countries, including most of the CPG organisations active in the published and grey literature on patient and public involvement. ${ }^{4} 2335$ Validation of findings against original data report by an international group of guideline developers and consumer representatives brings robustness to the findings.

On the other hand, practical constrains kept us from recording discussions, which would have strengthened data collection and analysis. Using the G-I-N conference workshop participants for convenience sampling also limits the consulted audience to persons likely to be supportive of both CPG and PPIP. Participants from developing regions and patient representatives were under-represented, partly because of financial barriers to attend. Previous studies found that patients can have a different view of their role in $\mathrm{CPG}^{8}$ and in our study, patient and public representatives highlighted the need for early and active involvement, as well as the potential that PPIP may improve the quality of guidelines. Other participants voiced concerns that PPIP may introduce controversy, bias, and resistance from professional groups, thus illustrating the delicate balance between the search for legitimacy and credibility among the different 'publics' involved in CPG development and use (health professionals, researchers, patients, policymakers, and the wider public). ${ }^{38-42}$

\section{CONCLUSION}

In recent years, CPG organisations have set up PPIPs that use a range of participation, consultation, and communication methods. A number of financial, organisational, and

Table 2 Priorities for patient and public involvement in guidelines identified by participants

\section{Research priorities}

1. To synthesise existing knowledge and experience in order to provide CPG developers with an overview of existing methods for involving patient and the public. Such synthesis should draw from the practical experience accumulated in other areas of healthcare (eg, research, health technology assessment, health policy, performance measurement).

2. To expand primary research on the pros and cons of different methods of involvement, including its impact on CPG development and implementation, as well as on CPG perceived validity, acceptability and legitimacy for health professionals, patients and the public. There is a need to study in greater detail the contextual and process factors that influence PPIP effectiveness.

3. To assess whether patient versions of CPG and information material foster informed and shared decision-making and impact on decisions. There is a need to understand how and in which situations patient decision aids and/or evidence on patient preferences and values should be integrated in existing CPG.

4. To adapt or expand the Appraisal of Guidelines for Research \& Evaluation criteria ${ }^{5}$ to better evaluate the quality of patient and public involvement in existing CPG.

International collaboration priorities

1. To develop recruitment methods, training and support strategies, information material and tools, and glossaries of technical terms used in CPG.

2. To develop common international standards and frameworks for PPIP development and evaluation that allow for adaptation to local context.

3. To support the development and exchange of information on alternative methods of patient and public involvement (eg, systematic reviews of evidence on patients' views and preferences and the integration of decision support tools).

4. To contribute to the enhancement of skills and expertise in countries with identified capacity needs.

5. To foster comparative research and evaluation of PPIP methods and impact on guideline development and implementation. 


\section{Key messages}

- CPG organisations are using a number of different methods to involve patient and the public in CPG development and implementation.

- The paucity of rigorous process and impact evaluations limits our current understanding of the conditions under which patient and public involvement programmes are most likely to be effective.

- Greater international collaboration is needed to strengthen existing knowledge, exchange experiences, and address barriers to effective involvement.

- Patient and public organisations and individuals need to be more closely involved in discussions over their role in CPG development and implementation.

socio-political barriers limit patient and public involvement in CPG. The lack of process and impact evaluations limits our current understanding of the conditions under which PPIP are most likely to be effective. Greater international collaboration is needed to strengthen existing knowledge, exchange experiences and expertise, and address barriers to effective involvement.

\begin{abstract}
Acknowledgements We would like to thank all workshop participants. Loes Knaapens provided important comments on the paper. G-I-N PUBLIC (http://www. ginpublic.net) is an official working group of the Guidelines International Network (G-I-N), an international not-for-profit association of organizations and individuals involved in the development and use of clinical practice guidelines, recognised under Scottish Charity Number SC034047. This paper reflects the views of the authors, and G-I-N is not liable for use that may be made of the information contained therein.
\end{abstract}

Funding $A B$ holds a joint doctoral scholarship from the Canadian Institute of Health Research (AnEIS programme) and the Agence de Santé et des Services Sociaux de l'Abitibi-Témiscamingue.

Competing interests None

Contributors $\mathrm{AB}, \mathrm{JB}, \mathrm{KC}, \mathrm{BF}, \mathrm{JG}, \mathrm{SS}, \mathrm{VT}$ and TVW organised the workshop, summarised discussions and, along with RG, contributed to the study design. MJ, JS and CS contributed to the interpretation of results. $A B$ drafted the paper and is its guarantor. All authors commented on the article for important intellectual content and approved the final paper.

Provenance and peer review Not commissioned; externally peer reviewed.

\section{REFERENCES}

1. Grimshaw JM, Thomas RE, MacLennan G, et al. Effectiveness and efficiency of guideline dissemination and implementation strategies. Health Technol Assess 2004:8:1-72.

2. Grol R, Wensing M, Eccles M. Improving patient care: the implementation of change in clinical practice. New York: Elsevier Butterworth Heinemann, 2005.

3. WHO. Global programme on evidence for health policy. Guidelines for WHO guidelines. Geneva: World Health Organization, 2003.

4. Schunemann HJ, Fretheim A, Oxman AD. Improving the use of research evidence in guideline development: 10. Integrating values and consumer involvement. Health Res Policy Syst 2006;4:22.

5. The AGREE collaboration. Appraisal of Guidelines for Research \& Evaluation (AGREE) instrument. 2001. http://www.agreetrust.org (accessed 10 Jul 2007).

6. Krahn M, Naglie G. The next step in guideline development: incorporating patient preferences. JAMA 2008;300:436-8.

7. Nilsen ES, Myrhaug HT, Johansen M, et al. Methods of consumer involvement in developing healthcare policy and research, clinical practice guidelines and patient information material. Issue 3. Cochrane Database Syst Rev 2006:CD004563. doi: 10.1002/14651858.CD004563.pub2

8. Boivin A, Green J, van der Meulen J, et al. Why consider patients' preferences? A discourse analysis of clinical practice guideline developers. Med Care 2009; $47: 908-15$

9. Ollenschlager G, Marshall C, Qureshi S, et al. Improving the quality of health care: using international collaboration to inform guideline programmes by founding the Guidelines International Network (GIN). Qual Saf Health Care 2004;13:455-60.
10. Buse K, Mays N, Walt G. Making health policy, London: Open University Press, 2006.

11. Rowe G, Frewer LJ. A typology of public engagement mechanisms. Sci Technol Human Values 2005:30:251.

12. Boivin A, Currie K, Burgers J, et al, eds. Patient and public involvement in guidelines (Part 1): Sharing international experiences. 5th International G-I-N Conference 1-3 October 2008, Helsinki: Finland

13. Boivin A, Burgers J, Fervers B, et a/on behalf of the Guideline International Network Patient and Public Involvement Working Group, eds. Patient and public involvement in guidelines (Part 2): Defining strategies for the future. 5th International G-I-N Conference 1-3 October 2008, Helsinki: Finland.

14. Green J, Thorogood N. Qualitative methods for health research. London: SAGE, 2004.

15. Gandjour A, Westenhofer J, Wirth A, et al. Development process of an evidencebased guideline for the treatment of obesity. Int J Qual Health Care 2001; 13:325-32

16. Raats CJ, van Veenendaal $\mathrm{H}$, Versluijs MM, et al. A generic tool for development of decision aids based on clinical practice guidelines. Patient Educ Couns 2008; 73:413-17

17. O'Connor AM, Legare F, Stacey D. Risk communication in practice: the contribution of decision aids. BMJ 2003;327:736-40.

18. Cabana MD, Rand CS, Powe NR, et al. Why don't physicians follow clinical practice guidelines?: a framework for improvement. JAMA 1999:282:1458-65.

19. Lugtenberg M, Zegers-van Schaick J, Westert G, et al. Why don't physicians adhere to guideline recommendations in practice? An analysis of barriers among Dutch general practitioners. Implement Sci 2009;4:54.

20. Burgers JS, Grol R, Klazinga NS, et al. Towards evidence-based clinical practice: an international survey of 18 clinical guideline programs. Int J Qual Health Care 2003;15:31-45.

21. Graham ID, Beardall S, Carter A0, et al. The state of the science and art of practice guidelines development, dissemination and evaluation in Canada. J Eval Clin Pract 2003;9:195-202

22. Carretier J, Bataillard A, Fervers B, et al, eds. From clinical practice guidelines to patient information: the French approach for patient involvement. 4th Guidleine International Network Conference 24 August 2007, Toronto: Canada.

23. van de Bovenkamp HM, Trappenburg MJ. Reconsidering patient participation in guideline development. Health Care Anal 2009;17:198-216.

24. Owens DK. Patient preferences and the development of practice guidelines. Spine 1998:23:1073-9.

25. Bastian $\mathbf{H}$. Raising the standard: practice guidelines and consumer participation. Int J Qual Health Care 1996;8:485-90.

26. McCormack JP, Loewen P. Adding 'value' to clinical practice guidelines. Can Fam Physician 2007;53:1326-7.

27. Nease RF Jr, Owens DK. A method for estimating the cost-effectiveness of incorporating patient preferences into practice guidelines. Med Decis Making 1994; 14:382-92

28. Dowie J, Wildman M. Choosing the surgical mortality threshold for high risk patients with stage la non-small cell lung cancer: insights from decision analysis. Thorax 2002:57:7-10

29. Sheridan SL, Harris RP, Woolf SH. Shared decision making about screening and chemoprevention. a suggested approach from the U.S. Preventive Services Task Force. Am J Prev Med 2004;26:56-66.

30. Mitton C, Smith N, Peacock S, et al. Public participation in health care priority setting: a scoping review. Health Policy 2009;91:219-28.

31. Thomas V. Involving patients and carers in developing national clinical practice guidelines - an evaluation. London: Open University, 2008.

32. Davies C, Wetherell M, Barnett E, et al. Citizens at the centre: deliberative participation in healthcare decisions. Sociol Health IIIn 2007:29:787-8.

33. Jarrett $\mathbf{L}$. A report on a study to evaluate patient/carer membership of the first NICE Guideline Development Groups. London: National Institute of Clinical Excellence Patient Involvement Unit, 2004.

34. Murphy MK, Black NA, Lamping DL, et al. Consensus development methods, and their use in clinical guideline development. London: Health Technology Assessment, 1998 Contract No.: 3.

35. Légaré $\mathbf{F}$, Boivin A, van der Weijden $\mathrm{T}$, et al. A knowledge synthesis of patient and public involvement in clinical practice guidelines: study protocol. Implement Sci 2009:4:30

36. Rosener JB. User-oriented evaluation: a new way to view citizen participation. J Appl Behav Sci 1981;17:583

37. Abelson J, Gauvin FP. Assessing the impacts of public participation: concepts, evidence and policy implications. 2006 http://www.cprn.com (accessed Feb 2009)

38. Kraemer JD, Gostin LO. Science, politics, and values: the politicization of professional practice guidelines. JAMA 2009;301:665-7.

39. Norheim OF. Healthcare rationing-are additional criteria needed for assessing evidence based clinical practice guidelines? BMJ 1999;319:1426-9.

40. Saarni SI, Gylling HA. Evidence based medicine guidelines: a solution to rationing or politics disguised as science? J Med Ethics 2004;30:171-5.

41. Chalmers I. The Alzheimer's Society, drug manufacturers, and public trust. BMJ 2007; 335:400.

42. Dyer $\mathbf{0}$. High court upholds NICE decision to limit treatments for Alzheimer's disease BMJ 2007;335:319. 